Silvia Brafman

\title{
Análise da variedade de produtos oferecida ao consumidor na indústria de cosméticos
}

Dissertação apresentada ao Programa de Pósgraduação em Engenharia de Produção da PUC-Rio como requisito parcial para obtenção do título de Mestre em Engenharia de Produção.

Orientador: Prof. Luiz Felipe Roris Rodriguez Scavarda do Carmo 
Silvia Brafman

\section{Análise da variedade de produtos oferecida ao consumidor na indústria de cosméticos}

Dissertação apresentada como requisito parcial para obtenção do título de Mestre (opção profissional) pelo Programa de Pós-Graduação em Engenharia de Produção da PUC-Rio. Aprovada pela Comissão Examinadora abaixo assinada.

Prof. Luiz Felipe Roris Rodriguez Scavarda do Carmo Orientador Departamento de Engenharia Industrial - PUC-Rio

Prof. Luiz Antonio Meirelles Departamento de Engenharia de Produção - UFRJ

Prof. Paulo Roberto Tavares Dalcol Departamento de Engenharia Industrial - PUC-Rio

Prof. José Eugênio Leal

Coordenador (a) Setorial do Centro Técnico Científico - PUC-Rio

Rio de Janeiro, 14 de setembro de 2009. 
Todos os direitos reservados. É proibida a reprodução total ou parcial do trabalho sem autorização da universidade, do autor e do orientador.

\section{Silvia Brafman}

Graduou-se em Administração de Empresas pela Universidade do Estado do Rio de Janeiro. Durante a graduação estagiou em empresas como Comissão de Valores Imobiliários (CVM) e Universal Music LTDA, em áreas ligadas a finanças. Depois de graduada ingressou em empresas de consultoria, primeiramente na Accenture do Brasil LTDA e, em seguida, na Webb Negócios S.A. Atualmente, trabalha na L'Oreal Brasil Comercial de Cosméticos LTDA.

Ficha Catalográfica

Brafman, Silvia

Análise da variedade de produtos oferecida ao consumidor na indústria de cosméticos / Silvia Brafman ; orientador: Luiz Felipe Roris Rodriguez Scavarda do Carmo. $-2009$.

87 f. : il. (col.); $30 \mathrm{~cm}$

Dissertação (Mestrado em Engenharia Industrial)Pontifícia Universidade Católica do Rio de Janeiro, Rio de Janeiro, 2009.

Inclui bibliografia

1. Engenharia Industrial - Teses. 2. Variedade de produtos. 3. Xampu. 4. Indústria de cosméticos. 5. Cadeia de suprimento. 6. Resposta eficiente ao consumidor. I. Carmo, Luiz Felipe Roris Rodriguez Scavarda do. II. Pontifícia Universidade Católica do Rio de Janeiro. Departamento de Engenharia Industrial. III. Título.

CDD: 658.5 
Dedico este trabalho aos meus pais, irmão e amigos que sempre me apoiaram e me deram a segurança para a realização dos meus sonhos. 


\section{Agradecimentos}

A toda minha família, em particular aos meus pais, Henrique e Doris, e irmão Leonardo, por todo o apoio, incentivo e compreensão durante a realização deste trabalho.

Ao meu Orientador Luiz Felipe Scavarda, pela atenção, dedicação, segurança, motivação e conhecimentos transmitidos durante a condução desta dissertação.

Ao Augusto da Cunha Reis, pelas observações e críticas essenciais para o desenvolvimento deste trabalho, atuando como se fosse um co-orientador.

Ao meu amigo e companheiro de trabalho, Fernando Ferreira, pela atenção a mim dedicada, paciência e ensinamentos.

Aos professores e funcionários do DEI da PUC-Rio, pelo apoio e infra-estrutura.

A todos os meus colegas de turma, que acompanharam de perto todo caminho percorrido durante o Mestrado, superando os desafios e comemorando comigo as vitórias conquistadas.

Aos meus grandes amigos, Aline, Paty, Marina, Dani, Patilene, Mari, Gus, Ka, Edu, Ro e a todos aqueles que de alguma forma contribuíram para a realização deste trabalho. 


\section{Resumo}

Brafman, Silvia; Carmo, Luiz Felipe Roris Rodriguez Scavarda do. Análise da variedade de produtos oferecida ao consumidor na indústria de cosméticos. Rio de Janeiro, 2009. 87 p. Dissertação de Mestrado Departamento de Engenharia Industrial, Pontifícia Universidade Católica do Rio de Janeiro.

A proliferação da variedade de produtos é uma tendência em diferentes indústrias e se constitui em um assunto com crescente relevância para o mundo industrial e acadêmico. A gestão dessa variedade deve buscar equilibrar os benefícios oriundos da perspectiva do marketing com os custos e obstáculos visualizados pelas perspectivas de operações e de logística. Assim, tal gestão se torna um grande desafio para as indústrias em que a variedade de produtos é elevada, como a de higiene pessoal, perfumes e cosméticos. A presente dissertação é um estudo empírico que tem como objetivo analisar esta gestão na indústria de cosméticos, quantificando, comparando e interpretando o comportamento da variedade de produtos oferecida ao mercado brasileiro, utilizando-se como objeto de estudo o xampu. Como variáveis desse produto, considerou-se divisão de mercado, grupo, grife, marca, finalidade adicional, público alvo, característica, cor, embalagem e fragrância. As análises da pesquisa foram realizadas a partir de uma pesquisa na indústria e de um estudo de caso em uma empresa multinacional no ramo de higiene pessoal, perfumes e cosméticos. Verifica-se como resultado da pesquisa que as variáveis marcas e características são as que mais impactam na geração de diversidade demonstrada ao consumidor e que não existe um comportamento padrão para a oferta de variedades de xampus. Já com o estudo de caso, por outro lado, pode-se concluir que a gestão da variedade de produtos está associada à gestão da cadeia de suprimentos (SCM) com intenso uso da técnica de resposta eficiente ao consumidor (ECR).

\section{Palavras-chave}

Variedade de produtos; Xampu; Indústria de Cosméticos; Cadeia de Suprimentos; Resposta Eficiente ao Consumidor. 


\section{Abstract}

Brafman, Silvia; Carmo, Luiz Felipe Roris Rodriguez Scavarda do. Analysis of the product variety offered to the consumer in the cosmetic industry. Rio de Janeiro, 2009. 87 p. MSc. Dissertation - Departamento de Engenharia Industrial, Pontifícia Universidade Católica do Rio de Janeiro.

Product variety is a trend in different industries and it is a subject with an increasing relevance both in the industry and academy. The management of product variety must balance the benefits obtained generally from the marketing perspective with the negative effects obtained, in general, from the operations and logistics perspective. Thus, it becomes a challenge for the industries in which product variety is high, such as the cosmetic industry. This master's dissertation is an empirical study that aims to analyze the management of product variety in this industry, quantifying, comparing and understanding the behavior of the variety of products offered in the Brazilian market, considering shampoo as the object of the study. As variables of this product it was considered market division, group, signature, brand, additional purpose, target audience, feature, color, packaging and fragrance. The analyses were done by an industry research and a case study in a multinational cosmetic company. It's verified as result of the industry research that the variables brand and characteristics are the ones that most contribute to generate diversity to the consumer. With the case study, on the other hand, it can be concluded that the management of this variety, is associated to the supply chain management (SCM) with intense use of Efficient Consumer Response (ECR).

\section{Keywords}

Product Variety; Shampoo; Cosmetic Industry; Supply Chain; Efficient Consumer Response. 


\section{Sumário}

1 INTRODUÇÃO 13

2 REVISÃO BIBLIOGRÁFICA 16

2.1 UM PANORAMA DOS ESTUDOS SOBRE A VARIEDADE DE PRODUTOS 16

2.2 DIMENSÕES E EVOLUÇÃO DA VARIEDADE DE PRODUTOS 21

2.3 PERSPECTIVAS SOBRE A VARIEDADE DE PRODUTOS 29

2.4 ESTRATÉGIAS DE MITIGAÇÃO 31

2.5 RESPOSTA EFICIENTE AO CONSUMIDOR - ECR 35

3 MÉTODO DE PESQUISA 40

3.1 OBJETO DE ESTUDO 40

3.2 DIMENSÕES DA VARIEDADE DE PRODUTOS PARA XAMPU 41

3.3 COLETA E PROCESSAMENTO DE DADOS - PESQUISA NA INDÚSTRIA 42

3.4 ColetA E PROCESSAMENTO DE DADOS - Estudo DE CASO 45

4 A INDÚSTRIA DE COSMÉTICOS 47

4.1 CARACTERIZAÇÃO DA INDÚSTRIA DE COSMÉTICOS 47

4.2 AS EMPRESAS DA PESQUISA 50

5 APRESENTAÇÃO E ANÁLISE DOS RESULTADOS DA PESQUISA NA INDÚSTRIA 54

5.1 ANÁLISE DA VARIEDADE ESTÁTICA 54

5.2 ANÁLISE DA VARIEDADE DINÂMICA 63

6 APRESENTAÇÃO E ANÁLISE DOS RESULTADOS DO ESTUDO DE CASO 67

7 CONCLUSÕES $\quad 76$

8 REFERÊNCIAS BIBLIOGRÁFICAS 80

$\begin{array}{ll}\text { APÊNDICE } & 86\end{array}$ 


\section{Lista de figuras}

FiguRA 1: NúMERO dE MOdELOS DE VEÍCULOS OFERECIDOS NO REINO UNIDO.

FIGURA 2: NÚMERO DE MODELOS DE VEÍCULOS PRODUZIDOS NO BRASIL. FIGURA 3: VARIEDADE DE COMPUTADORES EM UNIDADES ENTRE OS ANOS DE 1982 E 1992 NOS ESTADOS UNIDOS

FIGURA 4: VENDAS DURANTE A VIDA DO PRODUTO

FIGURA 5: CICLO DE VIDA DE UM PRODUTO

FIGURA 6: CONFIGURAÇÃO DO ECR

FIGURA 7: FATURAMENTO LÍQUIDO DA INDÚSTRIA QUÍMICA BRASILEIRA EM 2006

FIGURA 8: PARTICIPAÇÃO DOS SEGMENTOS NO FATURAMENTO DO SETOR 


\section{Lista de tabelas}

TABELA 1: ATRIBUTOS E VARIÁVEIS DA VARIEDADE DE PRODUTOS.

TABELA 2: COMPARAÇÃO DE VARIEDADE DE PRODUTO NA AMAZON.COM E EM UMA LOJA CONVENCIONAL

TABELA 3: DEFINIÇÕES PARA ECR

TABELA 4: ESTRATÉGIAS DO ECR, DEFINIICÕES E PRINCIPAIS ASPECTOS

TABELA 5: PRINCIPAIS PAÍSES EXPORTADORES DE PRODUTOS HPPC (EM US\$ MILHÕES)

TABELA 6: PRINCIPAIS PAÍSES IMPORTADORES DE PRODUTOS HPPC (EM US\$ MILHÕES)

48

TABELA 7: VARIEDADE DE XAMPUS PARA O MERCADO BRASILEIRO (ANO BASE 2008)

TABELA 8: VARIEDADE DE XAMPUS PARA O MERCADO FRANCÊS (ANO BASE 2008)

TABELA 9: VARIEDADE DE XAMPUS PARA O MERCADO BRASILEIRO (ANO BASE 1998) 


\section{Lista de equações}

EQUAÇÃO 1: CÁLCULO DA VARIEDADE DE PRODUTOS 


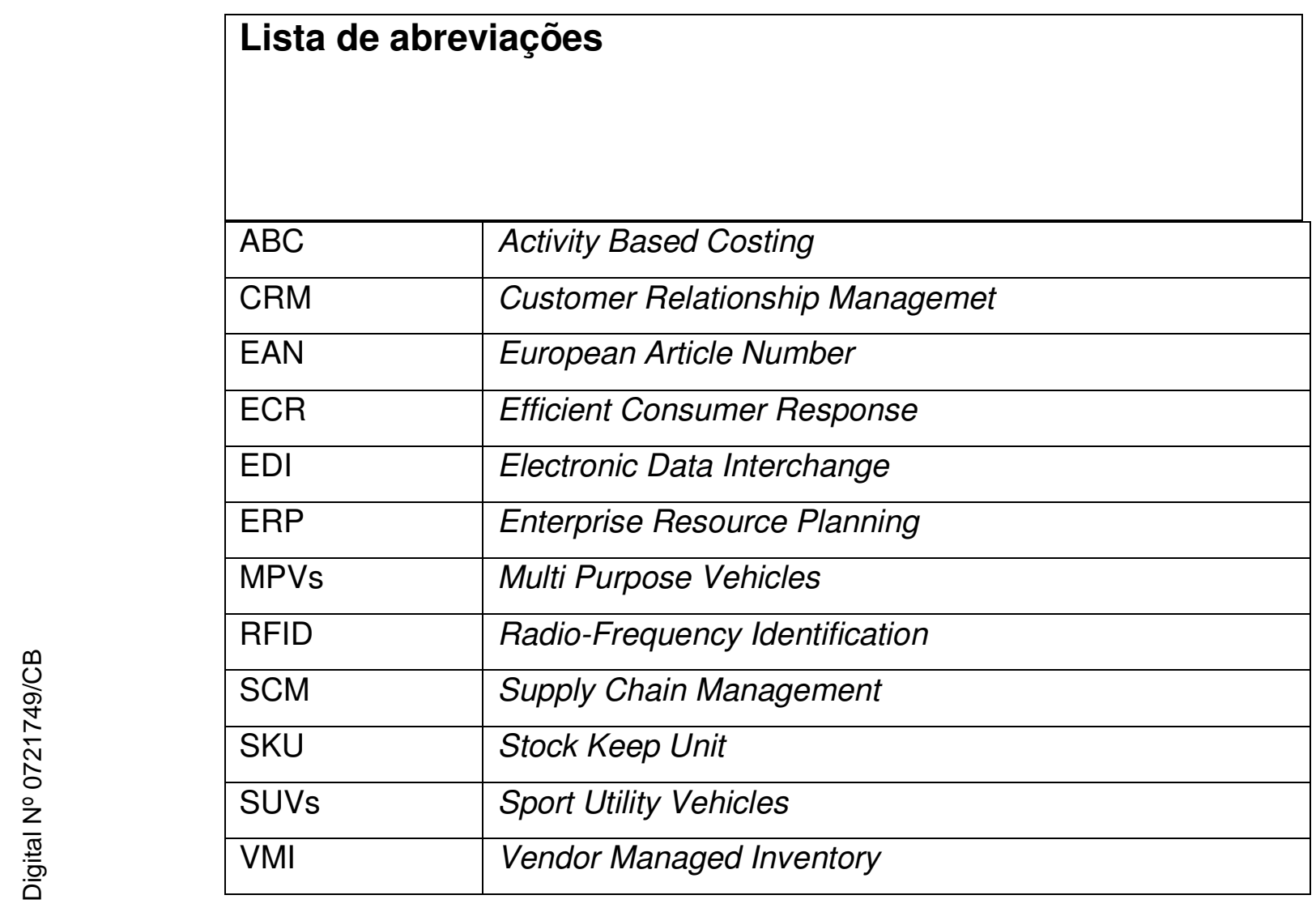

